\title{
Progress in ultra-lightweight glass ceramic space optics
}

\section{Roland Geyl, Eric Ruch}

Roland Geyl, Eric Ruch, "Progress in ultra-lightweight glass ceramic space optics," Proc. SPIE 11852, International Conference on Space Optics - ICSO 2020, 1185207 (11 June 2021); doi: 10.1117/12.2599147

SPIE Event: International Conference on Space Optics - ICSO 2021, 2021, Online Only 


\section{International Conference on Space Optics-ICSO 2020}

Virtual Conference

30 March-2 April 2021

Edited by Bruno Cugny, Zoran Sodnik, and Nikos Karafolas
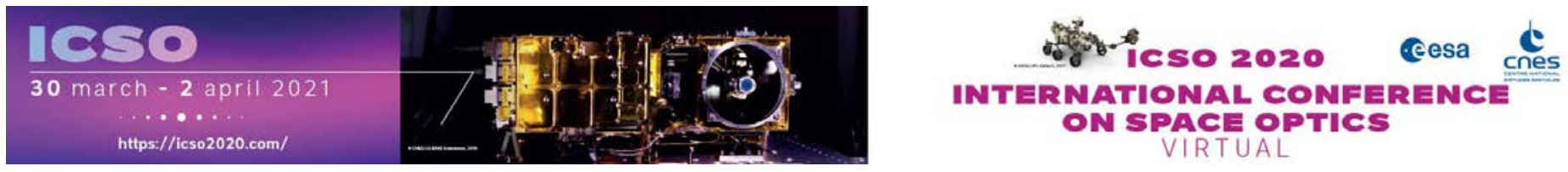

\section{Progress in ultra-lightweight glass ceramic space optics}

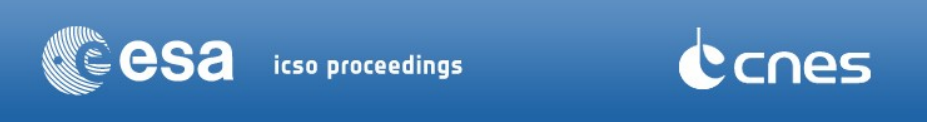




\title{
Progress in Ultra-Lightweight Glass Ceramic Space Optics
}

\author{
Roland GEYL, Eric RUCH \\ Safran Reosc, Avenue de la tour Maury, 91280 Saint Pierre du Perray, France
}

\begin{abstract}
Over more than four decades ago Safran Reosc was a pioneer in the development of lightweight glass ceramic space optics in Europe with FAUST, SPOT and METEOSAT projects for CNES. Since then we developed our best efforts to improve our skill and capabilities on the subject through many projects. We still hold a world record with the SOFIA primary mirror being the world largest machined lightweight glass ceramic mirror.

Despite our strong orientation toward the highly demanded Silicon Carbide material for space optical instrumentation Safran Reosc is still conducting high performance optical activities with glass ceramic material. Therefore, after recalling our solid heritage on the subject, we want to share with the community our recent developments in glassy ultralightweight optics with MERLIN, Kompsat-7 and thin shells for AO.

Other aspects of our progresses lie 1) in the improvements in term of schedule because the space industry is more and more schedule driven and 2) the evolution toward the use of fast and freeform optics enabling more capable and more compact, thus less heavy, and therefore lower total mission costs.
\end{abstract}

Keywords: space optics, lightweighting, FAUST, MERLIN, KOMPSAT-7, freeform, thin glass shell,

\section{THE PIONNEERING PROJECTS}

At Safran Reosc the skill of design and fabrication of lightweight space optics dates back up to the 70's. At this time we were the small company REOSC with a staff of about 30 people only specialized in large astronomical optics and instrumentation, with a solid capability on aspheric optics polishing. The CNES institution, created in 1961, was conducting its first developments in optical scientific instrumentation with the Laboratoire d'Astronomie Spatiale, now the Laboratoire d'Astrophysique de Marseille (LAM), mainly in the UV domain blocked by our atmosphere. REOSC was pleased to contribute to the Far UV Space Telescope (FAUST) UV camera ${ }^{[1]}$.

The design of this UV telescope was guided by simple considerations as follows:

Best optical throughput with min \# of mirrors with best UV coating reflectivity,

High detectivity with low F/\# to concentrate the maximum of light on the detector pixel,

Flat image surface for the UV light intensifier against which the film is pressed,

Wide Field of View larger than $7^{\circ}$ with good image quality.

The only possible design complying with all these requirements was the famous Wynne 2 mirror flat field anastigmat system with the M2 mirror larger than the M1 as show below.
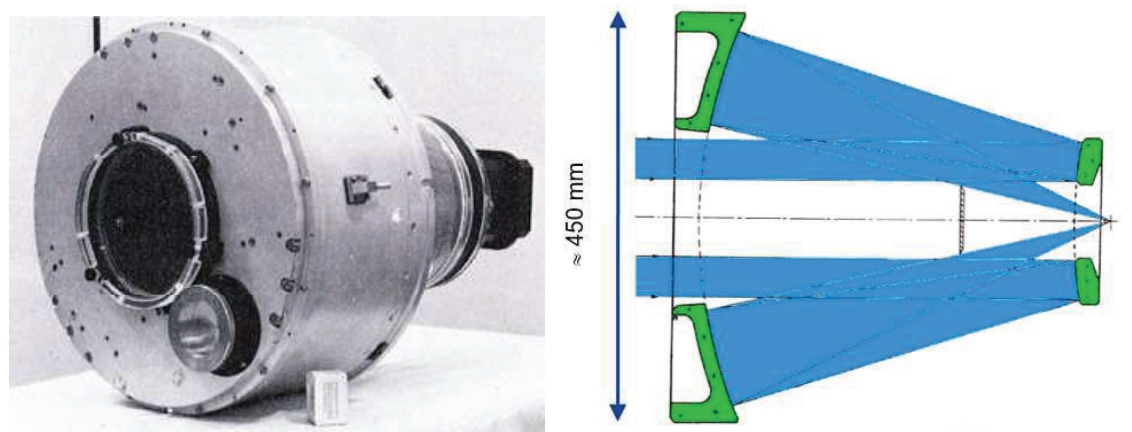

Figure 1. The FAUST instrument and its optical layout with the $\varnothing 450 \mathrm{~mm}$ lightweight secondary mirror. 
REOSC has been selected LAS for performing the optical manufacturing of the FAUST aspheric optics. Its large concave secondary mirror appears today as being the very first lightweight space optics delivered by the company. To our knowledge, this optics is probably also the first lightweight space optics made in Europe.

As seen on figure, the lightening profile of this mirror is very simple, designed without any Finite Element computation, and driven by the optical technician's abilities at that time. The material removal was done quite 'by hand' on a conventional grinder and thus limited to a large cylinder cavity from the rear and a following the front concave surface.

Logically such an optics was subject to gravity sag. Personally, I joined the REOSC Company in 1981 and I remember the comments of the control technician working on the project to the young engineer I was. He told me a few times that he clearly observed during Foucault knife-edge testing of the mirror under horizontal axis (no He-Ne interferometer at that time !) that the lightweight mirror was sagging under gravity due to the poor stiffness of the mirror body. When rotating the part on its two lateral support points he noticed that the deformation remained quite constant, thus meaning that it was not the result of a poor polishing of the optical surface but more a disturbing effect of the gravity. The FAUST project therefore was also the first one in Europe during which gravity effects on a lightweight optics were observed.

The FAUST instrument was flown several time onboard Veronique and Eridan rockets up to 1975 with interesting scientific returns. Later the instrument was selected by NASA for the Shuttle SpaceLab flight in 1992.

It seems also that a defense Earth Observation project was conducted during the same period. But we have not yet found solid data on these projects. It seems in fact that CNES stopped it for giving the priority to the civil projects of METEOSAT and SPOT offering direct return and benefits to the whole society.

For these two projects we introduced more elaborated lightening geometries and especially the semi closed-back structure. The main idea was to leave as much as possible of the rear plate contributing to the stiffness of the component during ground testing and to its strength during launch. The figure 2 below shows the lightweight optics produced for these two programs at the end of the 70's and early 80's.
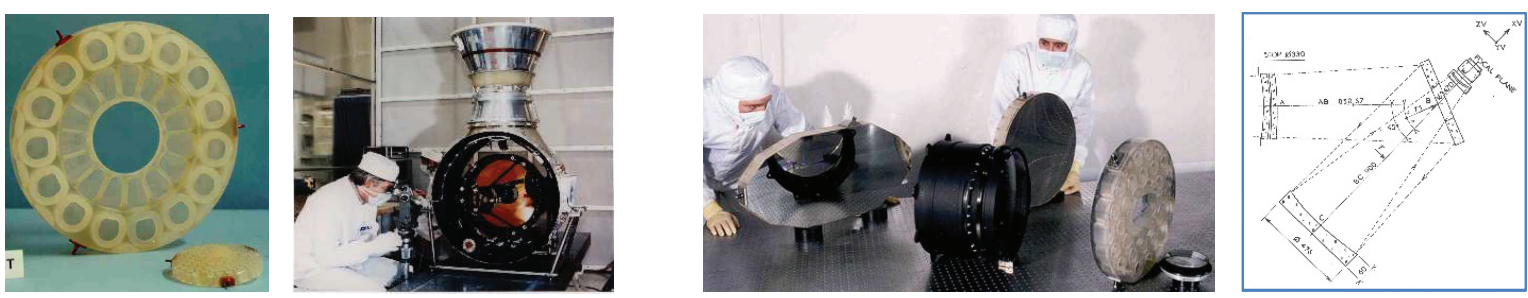

Figure 2. Left: the Meteosat $400 \mathrm{~mm}$ optics - Right : the SPOT-1 optics kit

\section{SOME MASTERPIECES AND RECORD}

In the domain of lightweight mirror made by glass-ceramic milling Safran Reosc produced several masterpieces and holds a world record we would like briefly to recall below.

\subsection{US-NAVY Sea Lite main mirror assembly}

We were awarded the contract from the US Navy for the development of the 1.8-m diameter main mirror assembly of the Sea Lite laser weapon onboard ships. The design is rather robust but mirror lightening was required for best turret agility. We machined semi-closed back pockets with $12 \mathrm{~mm}$ rib thickness through the $250 \mathrm{~mm}$ thick, 1.8 ton mirror body down to $450 \mathrm{~kg}$ only.

At the same time the mirror optical figure had to be preserved from gravity induced deformations along various orientations as well as the angular accelerations of the turret. For this purpose the mirror was installed on a 18 point stiff wiffle-tree support system.
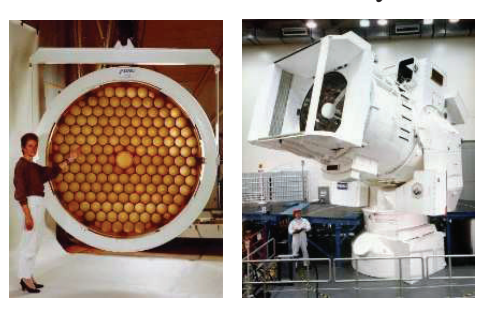

Figure 3. The Sea Lite lightweight mirror \& the laser beam director

After full success and customer satisfaction with the delivered unit, a second equipment was delivered a few years after. 


\subsection{The SOFIA primary mirror}

Another exciting project was the primary mirror for the Stratospheric Observatory for Infrared Astronomy (SOFIA). This instrument was a joint development between NASA in the US and DLR in Germany. To avoid the high costs of a space program like the Hubble Space Telescope the concept was then to install the telescope onboard a modified Boeing aircraft and to fly it at $16.000 \mathrm{~m}$ altitude, above the major part of the atmosphere and its humidity absorbing IR light. Another advantage of the concept is the possibility to update the system and change instrumentation after each flight.
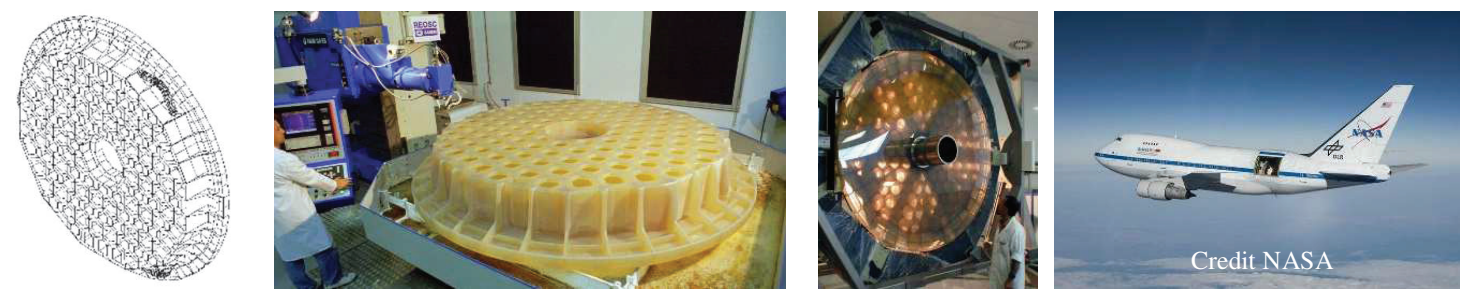

Figure 4. The SOFIA primary mirror design, machined, integrated, in flight

The main mirror has a huge size of 2.7-m diameter and the assembly was designed under similar considerations as for the Sea Lite mirror assembly, which served as a reference. Fortunately, an 18 points wiffle-tree support system was still sufficient to ensure the required quality under axial gravity. Laterally, bipods flexures helped to keep the mirror in place without distorting it. The mirror edge thickness was up to $390 \mathrm{~mm}$ and we drilled semi closed-back pockets combined with a large edge bevel during quite a year.

The SOFIA primary mirror still holds to our knowledge the world record of the largest glassy lightweight optics made by machining from a solid body ${ }^{[2]}$.

\subsection{Third GEMINI M2 Mirror}

Back to earth, we want to briefly introduce the GEMINI 8-m telescope project for which we developed a spare secondary mirror with exceptional quality, design and manufactured with space grade technology ${ }^{[3]}$. This $1-\mathrm{m}$ aperture convex mirror is installed on a tip-tilt chopping unit for image field stabilization at $100 \mathrm{~Hz}$ frequency and 2 arcmin amplitude. Low weight, low inertia, high strength and high optical quality are therefore required for this mirror, like for a space program. It also constitute the stop of the telescope and has to show smallest gap between optical and mechanical diameter. The figure 5 at right shows the open-back triangular geometry combined with a double arch profile to minimize gravity deformation under vertical optical axis.

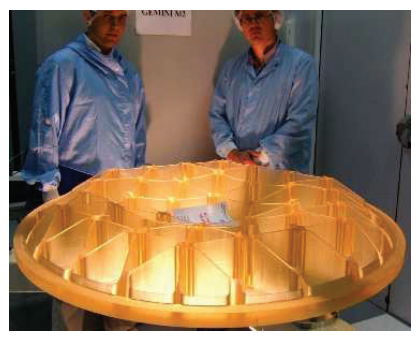

Figure 5. The GEMINI M2 mirror

This ground astronomical project was one where Safran Reosc performed significant improvements on its lightening technologies, directly applicable for space applications:

Optimizing machining process toward thinner ribs and less risks ( $5 \mathrm{~mm}$ over 150x150 $\mathrm{mm}$ rib area)

Refined more uniform and deeper acid etch up to $0,6 \mathrm{~mm}$ thickness to gain further mass

Bottom of pockets following accurately the optical surface curvature to gain mass and minimize quilting effect

Filet radii adjusted to reduce local stress level under dynamic solicitations

Controlling the polishing up to the very edge aperture thanks to IBF finishing

The optics ended very successfully with only $47 \mathrm{~kg}$ mass and $18 \mathrm{~nm}$ RMS reflected WFE optical quality.

\subsection{The SUNRISE telescope main mirror}

Sunrise is a balloon-borne solar observatory based on a $1 \mathrm{~m}$ aperture Gregory telescope, a project led by the Max Planck Institute for Solar System Research (MPS). Like for SOFIA installed within an airplane, using a balloon capable to fly up to $35 \mathrm{~km}$ altitude is a way to approach the attractive space conditions for scientific instrumentation without the cost of a satellite. The optical requirements for the main mirror remain at the level of the space standards and ask for an ultralightweight $1 \mathrm{~m}$ class primary mirror, facing the sun and thus collecting about $1 \mathrm{~kW}$ of optical energy. 
The Silicon Carbide was originally planned for this mirror. But budget reasons and insufficient technology readiness of $\mathrm{SiC}$ led Safran Reosc to propose Zerodur glass ceramic from Schott for the main mirror. Zerodur is balancing its low thermal diffusivity by a very low coefficient of thermal expansion, thus ensuring the preservation of the high optical quality of the front surface mirror under thermally perturbed environment under the balloon.

Safran Reosc designed and manufactured this $1 \mathrm{~m}$ mirror down to a mass of $45 \mathrm{~kg}$ only but with an original open-back design with $3,5 \mathrm{~mm}$ only rib thickness and a large trefoil profile on its rear side. This design has been optimized to maximize the strength of the component and minimize the deformation of the front surface under gravity acting laterally, i.e. when the telescope is pointing near the horizon. Today the Sunrise telescope already performed three flights and, each time, its primary mirror has been safely recovered despite sometime tough landing conditions.
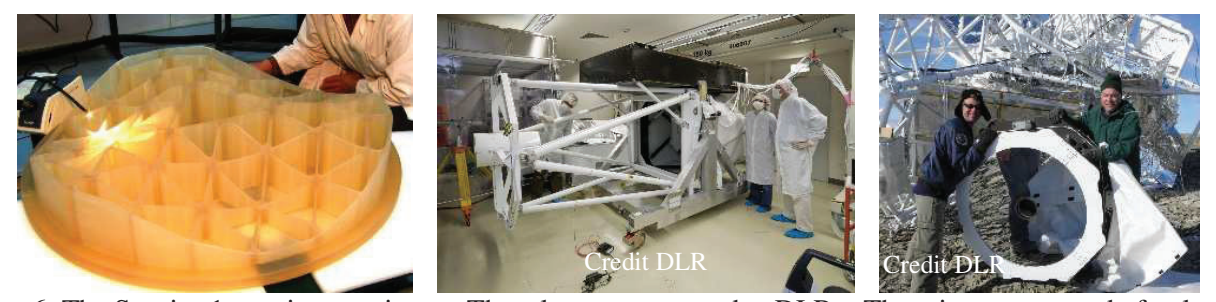

Figure 6. The Sunrise $1 \mathrm{~m}$ primary mirror - The telescope prepared at DLR - The mirror recovered after landing

\section{OPTIMIZING THE PROCESS}

Through the decades of efforts on the subject of lightweight optics for space, science or defense our team has continuously improved the technology under the various aspects of design, manufacturing, polishing as well as testing. These all subjects remain in fact more or less interconnected and a global vision remains necessary.

\subsection{Optimizing lightweight mirror design}

The initial lightweight design originating from FAUST M2 mirror conducted us to plan to adopt for the first generation of SPOT and Meteosat mirrors a network of such cylindrical pockets simply machined from the rear. However, it quickly appeared that the efficiency of this design is poor.

We then moved to the semi-closed back structure with T-shaped tools allowing doing undercutting and thus leaving a part of the rear plate that contributes well to the overall stiffness of the mirror body. A further refinement was then to drill small holes in the thicker area between the various large cylinders.

With more elaborated NC machines coming on the market, hexagonal machining has been used successfully to generate constant thickness of the walls separating the various pockets. However the radius of the top of the T-shaped grinding tools is leaving rather large filet radii between the walls. On the bottom of the pockets small filet radii offer a smooth transition between the front optical surface plate and the vertical walls for lower local stresses during high mechanical solicitations during launch.

A step has been taken with the move to open back structure with triangular cells. These offer the advantage of straight ribs all over the body's diameter offering higher global stiffness of the structure. Machining risks are lower also with this concept. However, the height of the ribs must be increased to compensate the absence of rear plate. In fact there is always a trade-off to be done between the last designs, depending the targeted lightweight ratio, mirror size, etc...
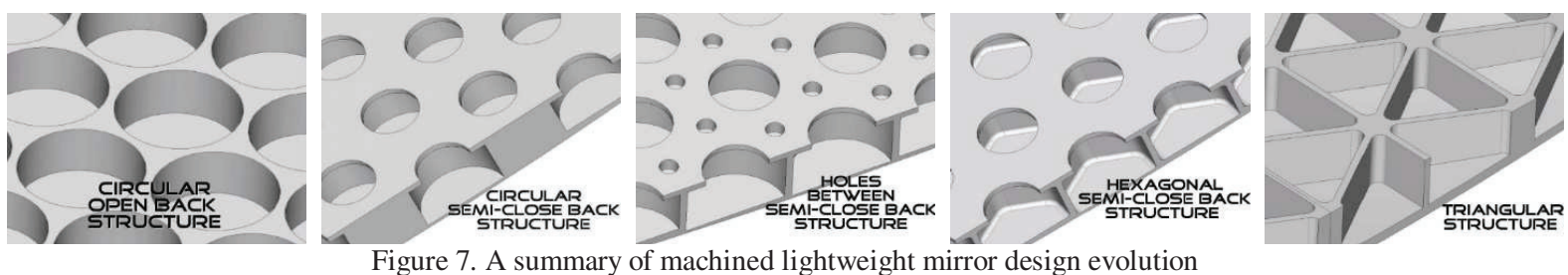

Figure 7. A summary of machined lightweight mirror design evolution 
Another refinement was adopted for the first time for the Gemini M2 mirror project with the bottom of the pockets following precisely the curvature of the optical surface. Of course, this is more easily achievable with an open-back structure allowing the tool to scan the whole bottom of the lightweighting pocket. The main advantage of this is that the facesheet thickness can be machined well uniform, contributing to lower quilting effect during polishing and/or some gain of mass at this level. Figure 8 below shows the concept and a real example.
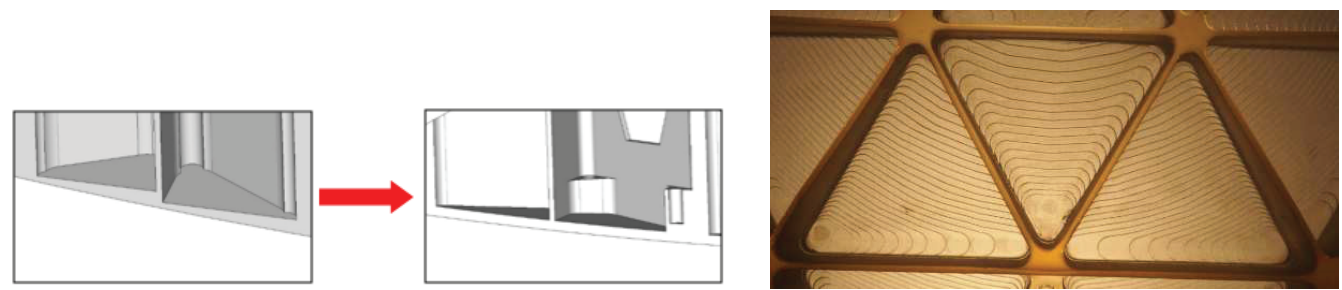

Figure 8. Evolving towards front facesheet of uniform thickness

\subsection{Optimizing the manufacturing}

We will not hide that we sometime broke a piece during the delicate machining operations. However, each damage constitutes an opportunity for learning and improving the technique. We cannot enter into too much details but there are many factors on which great attention must be taken.

The tool itself must be made from appropriate material onto which diamond pellets and rings have to be brazed with the best quality.

As the operation are rather long, with lateral efforts on the tool spindle, the design and control of the fatigue of the tools has to be accurately addressed.

The machining parameters, spindle speed and tool feed, must be optimized with respect to the type of machining you are doing.

The machining strategy itself, what to drill first, what to do in the last steps, is also important as there is always one such strategy that induces less stress in the part and less risk of breakage.

Finally, automation of the machining center for 24/7 operation is useful to gain schedule. However, the detection of abnormal conditions, by a human operator or some adequate sensors is mandatory to secure the process.

Safran Reosc has been developing many efforts to progress into the direction of lower mass, larger mirrors and higher light-weighting ratio. Demonstration work ${ }^{[4]}$ of aggressive light-weighting have been reported with area densities of 35 $\mathrm{kg} / \mathrm{m}^{2}$ for large aperture mirrors thanks to $2.5 \mathrm{~mm}$ only rib thickness, $5 \mathrm{~mm}$ facesheet thickness and $6 \mathrm{~mm}$ small radius between walls. With additional acid etching rib thickness below $2 \mathrm{~mm}$ are achievable. See the $500 \mathrm{~mm}$ demonstration part on figure 9 below.

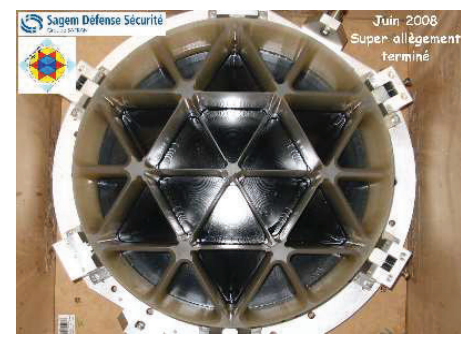

Figure 9. Ultra lightweighting breadboarding activities

\subsection{Optimizing the polishing}

Robotic polishing: Safran Reosc has jumped into robotic polishing quite 30 years ago in the early 90's. The use of robot immediately helped us not to be bothered by cinematic issues, like for a gantry system, and to have a full freedom of movements at our disposal. Therefore we were able to concentrate our efforts on the polishing process itself rather than on the mechanical problems. This choice was more than successful and were able to develop and mature our technology vary rapidly for axisymmetric, off-axis and ... freeform optics. 
Ion Beam Figuring: This technique originates from the semiconductor industry. It consists in Argon ions projected under vacuum onto the optical surface with the appropriate energy which the remove material at atomic level with high accuracy. As it is a non-contact process, IBF is well suited for the removal of residual quilting from contact pitch polishing as well as the reduction of edge effect. Combined with its high accuracy and correct removal rate IBF it is a productive tool for finishing ultra-lightweight optics. At Safran Reosc we have now several IBF equipment dedicated to small size, medium size and large optics up to 2.5-m.
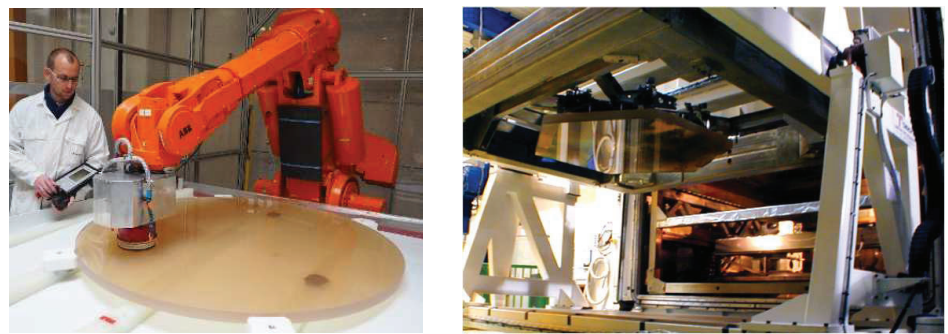

Figure 10. Large optics robotic polishing and IBF finishing equipment.

Toward freeform optics: Freeform optics are now appearing within precision optical instrumentation for imagery or spectroscopy and offer the key advantage of either best compactness for the same performance level, better performances for the same volume of the optics, or a mix of both advantages.

This is more and more essential for space instrumentation as gain on the optical instrument volume induces gain on its weight and inertia and therefore gain on the platform itself volume and weight as well as the launch cost. At the end there appear a significant gain on the total mission cost thanks to the introduction of freefrom optics. In summary the SWaP factor (Size, Weight \& Power) is key and freeform optics enable a quantum jump on the subject ${ }^{[5],[6]}$.

At Safran Reosc we immediately understood the huge benefit that freeform optics can offer and we invested of this technique. In fact, this was easy as our robotic and IBF technologies were already fully freeform. We have been selected by Airbus for the pioneering Microcarb instrument, the first one in Europe including five high performance freeform mirrors. After our delivery of the full set of optics we were pleased to learn that Airbus was able to align them successfully (not an easy task too! ) and to obtain the targeted image quality: a premiere in Europe.

Today we conduct several line of actions with CNES on the subject of freeform optics. One of these is on the design side with the elaboration of new concepts of high capability space telescope fitting in the most compact volume.

Another one is on the demonstration of the optical manufacturing capability of twice time more aggressive than Microcarb freeform optics. For this, a demonstration piece was produced recently with the following features:

Size

: $250 \times 150 \mathrm{~mm}$

Freeform profile : 36 Zernike terms

Amplitude : : max departure from best sphere of 4,2 $\mathrm{mm}$

Slopes : $\quad$ max slope vs best sphere of $55 \mathrm{mrad}$

Achieved quality : < $14 \mathrm{~nm}$ RMS residual surface error
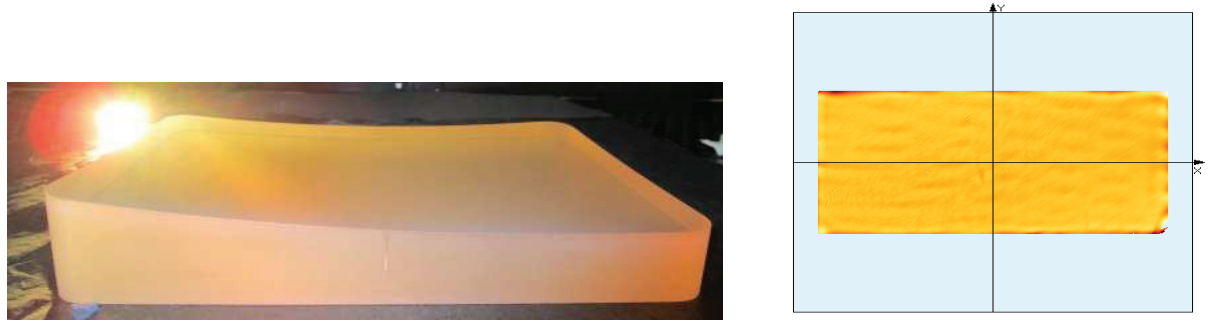

Figure 11. Severe freefrom polishing demonstration to high performance (14 $\mathrm{nm}$ RMS residual surface error) 


\subsection{Optimizing the testing}

Strong efforts are continuously developed to improve the industrial efficiency of our metrology. Our metrology team develop new concepts and bring the instrument to a real operational level before transferring it to the production team. The main tools are optimized for the various steps of optical fabrication:

3D Coordinates Measuring Machines (CMM), used in the early stages of the manufacturing process: milling, grinding and lapping operations.

In the last steps Computer Generated Holograms (CGH), used to evaluate the surface deviations from ideal profile with nanometer accuracy. The $\mathrm{CGH}$ alignment is now quite automatic for quick turnover of the various optical elements passing on an universal test bench.

The in-between steps of fabrication are often the called the 'death valley' because 3D CMM is not accurate enough and the optical part figure error and slope error are too high for precision interferometric evaluation with a CGH. Here deflectometry is well filling the gap thanks to its various advantages: it is non-contact, offers high spatial resolution with a large dynamic range and shows good sensitivity to mid spatial frequency errors.

A last point is the determination of the gravity sag. We perform this by combining appropriately several measurement with a proprietary methodology in order to extract from them, as accurately as possible, the contribution of the gravity effect. The larger the optics the more important this effect is becoming, either under vertical optical axis or horizontal optics axis.

Figure 12. Gravity effect
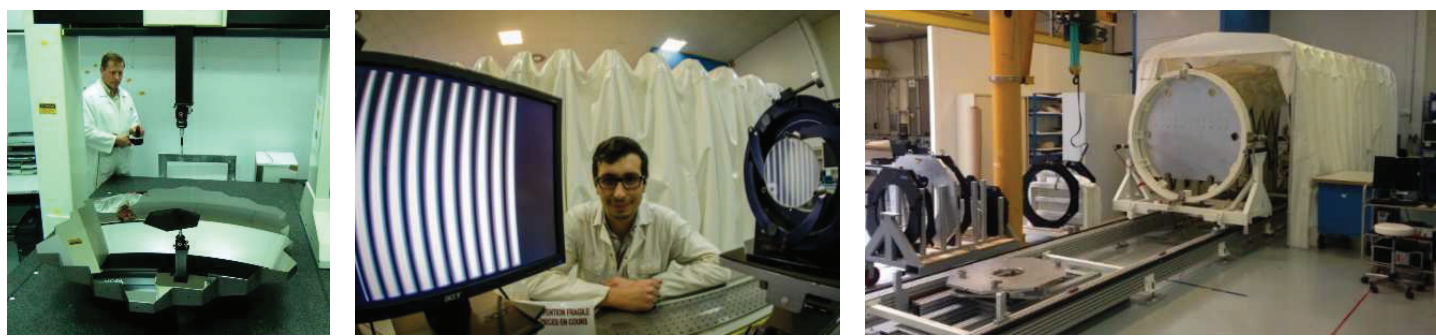

Figure 13. Optimized optical metrology: 3D CMM - Deflectometry - CHG bench

\section{TWO RECENT ADVANCED PROJECTS}

We want to report now two recent projects that highlight our progress and master of the skill for lightweight space optics made from glassy material. The first one, MERLIN Lidar optics, is a state of the art ultra-lightweight, ultra-aspheric mirror. The second one, Kompsat-7 is a large precision mirror differentiating by the tight schedule under which its development is to be conducted.

\subsection{MERLIN RX telescope primary mirror}

MERLIN, the Methane Remote Sensing LIdar MissioN, is a Franco-German collaborative minisatellite climate mission shared between the French Space Agency CNES and the German Space Administration DLR ${ }^{\text {[7] }}$. The primary objective is to obtain spatial and temporal gradients of atmospheric methane $(\mathrm{CH} 4)$ columns with high precision and unprecedented accuracy on a global scale. It has to be known that Methane is $25 \mathrm{x}$ more contributing to greenhouse effect than $\mathrm{CO} 2$, so its presence in our atmosphere must also be studied in detail.

CNES is supplying the Myriade Evolution platform and DLR has awarded the contract for the instrument to Airbus Defense and Space GmbH. The instrument is an Integrated Path Differential Absorption LIDAR (IPDA). At the begin of the development, the main driver for the actual payload design were the limited payload allocations by the platform: a maximum mass of about $95 \mathrm{~kg}$ in a volume of $82 \mathrm{~cm} \mathrm{x} 88 \mathrm{~cm}$ x $92 \mathrm{~cm}$. While the mass could be relaxed, the volume and the limited M1-M2 mirror distance remained.

Safran Reosc has been selected for the laser emitting optics and the receiver telescope. To cope with the SWaP constraints of the Myriade Evolution platform Airbus selected a very compact design with the whole instrument fitting within a cube as shown on the figure 13 below. 
The key challenge for the receiver telescope is its main mirror which is an ultra-fast off-axis parabola with the following main characteristics:

$\begin{array}{ll}\text { Optical design entrance pupil } & \text { Ellipse of } 732 \times 690 \mathrm{~mm} \\ \text { Off-axis distance } & 410 \mathrm{~mm} \\ \text { Mechanical diameter } & 745 \mathrm{~mm} \\ \text { Parent mirror aperture } & \varnothing 1512 \mathrm{~mm} \\ \text { Focal length } & 575 \mathrm{~mm} \\ \text { Parent mirror F/\# } & \text { F/0.38 } \\ \text { Aspheric departure } & 16 \mathrm{~mm} \\ \text { Polishing specification } & 50 \mathrm{~nm} \text { RMS residual surface error }\end{array}$

In addition, the lowest possible weight was requested for this mirror. We therefore applied the various techniques developed during our previous work on ultra-lightweight mirror and designed an aggressive mirror geometry. Starting from a $745 \mathrm{~mm}$ diameter x $97 \mathrm{~mm}$ glass blank of $107 \mathrm{~kg}$ mass, we ended to a final mass of 11,9 $\mathrm{kg}$ only with $3 \mathrm{~mm}$ thin ribs, thus a lightening percentage close to $90 \%$ and an areal density below $25 \mathrm{~kg} / \mathrm{m}^{2}$.
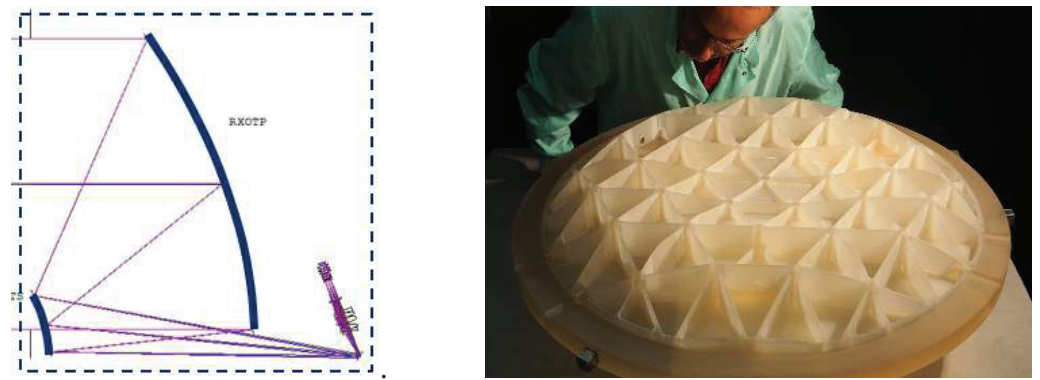

Figure 13. MERLIN receiver telescope optical sketch and primary mirror

At the time of writing this manuscript, the Qualification Model (QM) of the receiver telescope main mirror has been equipped with its pads and flexures and is undergoing its final IBF run toward the optical specification. We will then proceed to the mechanical and thermal qualification tests and will then be able to deliver this model. The Flight Model (FM) has been lightweighted too and it first polishing step is close to specification. Before equipping it with pads and flexures and finalizing its polishing operation we have to wait the results of the QM qualification sequence.

\subsection{Kompsat-7 primary mirror}

South Korea is very active in earth observation from space. The Korea Aerospace Research Institute (KARI) is developing cutting-edge satellites for observing the earth, especially the serie of Korea Multi-Purpose Satellite (KOMPSAT - Arirang). The latest generation is Kompsat-7 that will offer world-top class resolution and is presently under development.

Safran Reosc is proud to have been selected for the contract for developing the main telescope optics kit, and especially its main mirror. KARI pushed us to perform this development in the shortest schedule and we are proud to have achieved this task rather well thanks to the full maturation of our panel of technologies and the full dedication of our team.

The main mirror is lightweighted in an open back configuration with an areal density below $80 \mathrm{~kg} / \mathrm{m}^{2}$
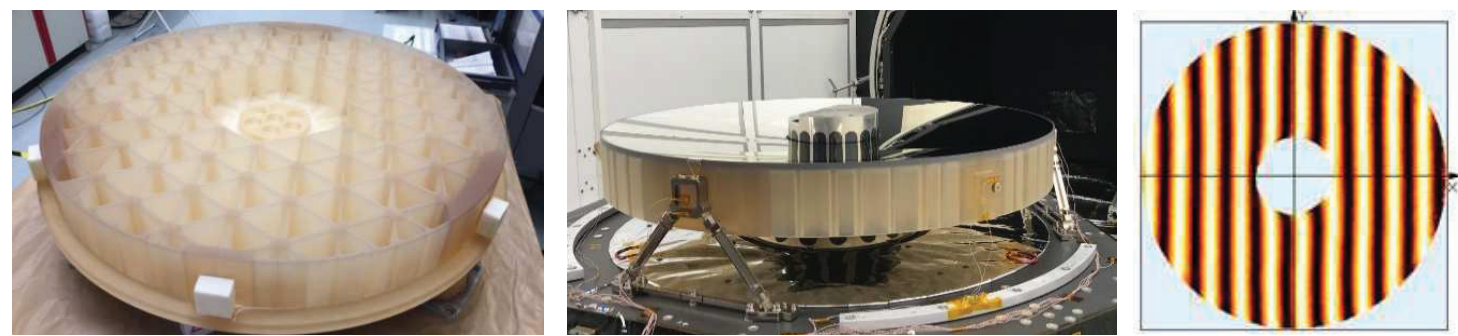

Figure 14. Kompsat-7 main mirror lightweighted, integrated on its support frame, final simulated 0g fringe pattern (12 nm RMS WFE) 
At the time of writing this manuscript, the Qualification Model (QM) has already been delivered. The Flight Model \#1 (FM1) is under final acceptance and will be delivered soon. The FM2 is progressing very well and is planned for delivery this year.

We see that Safran Reosc is able to deliver such state of the art space optics to the unpreceded rate of about one unit every 6 months. Knowing that the space business is today more and more schedule driven rather than just performance driven, we are proud to report such solid industrial capability on the difficult activity of large lightweight precision space optics designing, lightening, polishing, integrating and testing.

\section{THIN GLASS SHELLS}

Another version of ultra-lightweight large space optics is the case of thin shells used in active mode. The stiff, but heavy rigid mirror body, is here replaced by a thin shell actively controlled in shape and figure by several actuators distributed over a reference plate.

This technique is more and more used today with full success in the domain of ground astronomy for adaptive correction of the high temporal frequency effects of the atmospheric turbulences. In space, the concept can be used in active mode, i.e. slow temporal correction of the deformation of the optical surface. Several studies have been conducted in Europe some years ago and more recently according to various active optics concepts and strategies, but most of them include large thin shells.

For space applications we would like to recall that we pioneered the research with a $80 \mathrm{~cm}$ diameter concave shell mirror of $5 \mathrm{~mm}$ thickness only in 1988 made in the frame of an ESA study. Later, we delivered in 1995 a 1.6-m aperture x 3 $\mathrm{mm}$ thickness hexagonal thin shell to Composite Optics (now ATK) in the frame of technological development research for the JWST primary segment, based on the technology of such thin shell bonded on a CFRP backing structure.

In the domain of ground astronomy Safran Reosc has delivered successfully such type of optical components for the European Southern Observatory (ESO) Very Large Telescope secondary mirror Adaptive Optics Unit: the VLT M2 AO Unit ${ }^{[8]}$. This is the replacement of one of the original solid Beryllium secondary mirrors, also supplied by Safran Reosc, by its full Adaptive Optics version. We produced a $1 \mathrm{~m}$ diameter, convex aspheric thin glass shell of $2 \mathrm{~mm}$ thickness only with $15 \mu \mathrm{m}$ thickness uniformity. The VLT M2 AO Unit is now fully operational on-site in Chile. Today we are also close to deliver the first set of 6 petal-shaped thin shells for the Extremely Large Telescope M4 AO Mirror Unit.
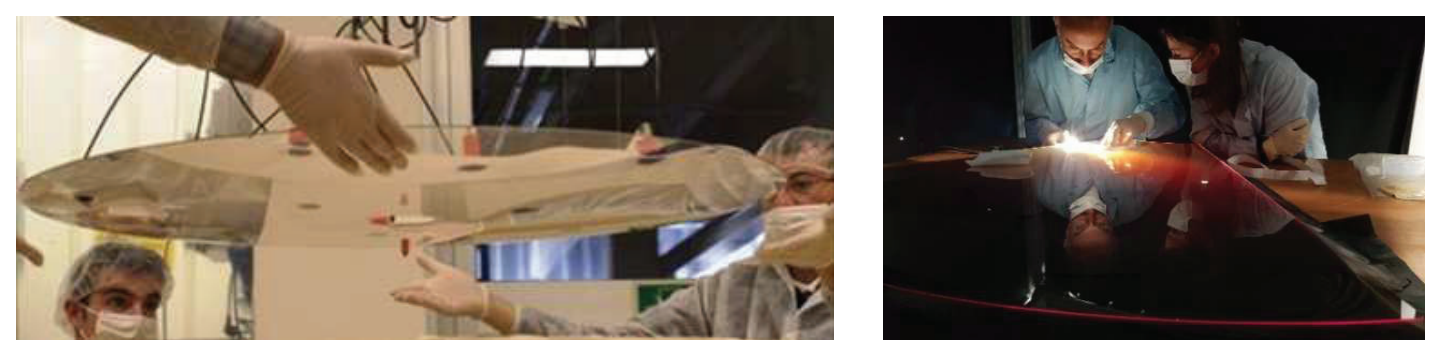

Figure 15. Left: Thin glass shell for VLT M2 AO Unit - Right: The first petal shaped thin shell for ELT M4 Unit
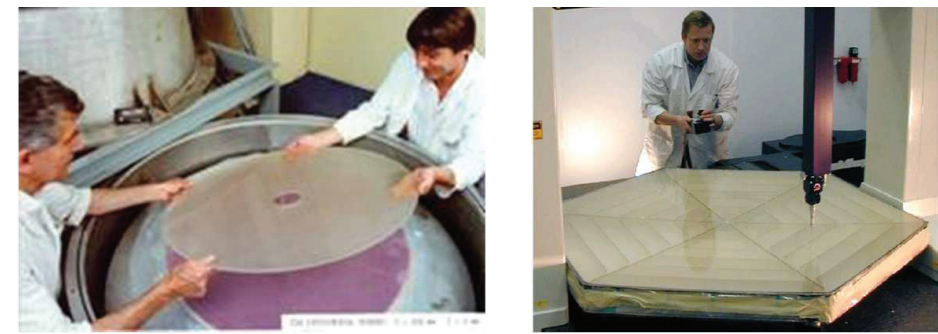

Figure 16. Left: Semi-passive mirror for ESA study and Right: Hybrid mirror with thin shell on CFRP structure 


\section{CONCLUSION}

After pioneering activities on lightweight space mirror made by machining a glass-ceramic mirror body backing in the 70's with the FAUST UV telescope, Safran Reosc has contributed to the first key space programs SPOT and METEOSAT with continuously improved mirror technology.

Our world record 2.7 meter SOFIA primary mirror is flying now routinely with a specially modified Boeing aircraft at 16.000 meter altitude, thus approaching space conditions for IR astronomy. The SUNRISE telescope on board a balloon includes a high-tech primary mirror developed under an original design allowing it to be as efficient as the Silicon Carbide concept originally considered.

Beside our dedication to precision Silicon Carbide mirror polishing, we never stopped to improve our art and skill for designing, machining, polishing, testing and integrating larger and larger and always more precise lightweight glassy mirrors.

Recently our skill has been applied to the development of the MERLIN receiver telescope ultra-lightweight and fast primary mirror enabling to install this instrument on board the Myriade Evolution smallsat platform, thus enabling much cost saving at system level for this mission.

Industrially, Safran Reosc is also showing with Kompsat-7 project its capability to deliver precision large space optics to unique performance level and high production rate. Space is continuously evolving towards faster, better and cheaper and we do our best to cope with this market demand.

Ahead of us, the use of active optics will soon come for innovative space optical instrumentation. Here we has raised to TRL 9 our technique for producing large thin glass shells. Such shells are fully operational within the VLT ground telescope and production of shells for the Extremely Large Telescope is underway.

We thank CNES for pushing the REOSC Company in this direction of space optics and want to thank all our employees who contributed through the years and today to the level of skill we have reached today on the subject of lightweight glass-ceramic mirrors.

\section{REFERENCES}

[1] J.M. Deharveng \& al, Laboratoire d'Astronomie Spatiale, "FAUST Instrument: A high focal ratio telescope for Far and Near UV imagery", Space Science Instrumentation 5, 1979.

[2] R. Geyl \& al, Sagem Reosc, "SOFIA Primary Mirror Design, Fabrication \& Testing", SPIE Vol 4451, Optical Manufacturing \& Testing IV, San Diego, 2001. https://doi.org/10.1117/12.453610

[3] R. Geyl \& al, Safran Reosc, "Progress toward a third Gemini secondary mirror", SPIE Vol 5869, Optical Manufacturing and Testing VI; San Diego, 2005. https://doi.org/10.1117/12.621500

[4] E. RUCH \& al, Safran Reosc, "Highly light-weighted Zerodur mirrors", International Conference on Space Optics, Toulouse, 2008

[5] R. Geyl, Safran Reosc, "Advanced space optics development in freeform optics design, ceramic polishing, rapid and extreme freeform polishing", International Conference on Space Optics (ICSO) 2016.

[6] R. Geyl \& al, "Freeform optics technologies for space", International Conference on Space Optics (ICSO), Chania - Greece, 2018

[7] Ch. Wührer \& al, "MERLIN: Overview of the design status of the Lidar Instrument", International Conference on Space Optics (ICSO), Chania - Greece, 2018

[8] R. Geyl \& al, "Thin glass shells, a new type of optical components", European Optical Society - Manufacturing of Optical Components, Munich, 2013. 